\title{
Diffuse Cerebral Vasoconstriction in a Intravascular Lymphoma Patient with a High Serum MPO-ANCA Level
}

\author{
Atsuhiko Sugiyama ${ }^{1,2}$, Makoto Kobayashi ${ }^{1}$, Ayaka Daizo ${ }^{3}$, Miyako Suzuki ${ }^{3}$, \\ Hirotoshi Kawashima ${ }^{3}$, Shin-ichiro Kagami ${ }^{3}$, Hiroaki Tanaka ${ }^{4}$, Yoshio Suzuki ${ }^{5}$, \\ Takashi Matsunaga ${ }^{1}$ and Satoshi Kuwabara ${ }^{2}$
}

\begin{abstract}
An 87-year-old woman presented with a 3-month history of fever, edema of the lower legs, and gait disturbance. A laboratory examination revealed high serum levels of myeloperoxidase-specific antineutrophil cytoplasmic antibody (MPO-ANCA). Although microscopic polyangiitis was initially suspected and treated, the patient subsequently developed transient hemiparesis and disturbed consciousness. Brain magnetic resonance imaging/angiography revealed infarct-like lesions, pachymeningeal involvement, and diffuse cerebral vasoconstriction. A random skin biopsy confirmed the histological diagnosis of intravascular lymphoma. Diffuse cerebral vasoconstriction and a high serum MPO-ANCA level have rarely been reported in patients with intravascular lymphoma. Endothelial damage due to immune-mediated mechanisms, tumor derived factors, or the direct interaction of lymphoma cells with endothelial cells may commonly predispose patients to both cerebral vasoconstriction and the development of ANCAs.
\end{abstract}

Key words: intravascular lymphoma, vasoconstriction, magnetic resonance angiography, myeloperoxidasespecific antineutrophil cytoplasmic antibody

(Intern Med 56: 1715-1718, 2017)

(DOI: 10.2169/internalmedicine.56.8051)

\section{Introduction}

Intravascular lymphoma (IVL) is a rare type of extranodal lymphoma with an aggressive clinical course. It is characterized by a propensity for the proliferation of lymphoma cells within the lumina of small vessels with a predilection for the central nervous system and skin (1). Regarding the central nervous system involvement, several characteristic findings of brain magnetic resonance imaging (MRI) have been reported, including infarct-like lesions, dural/meningeal enhancement, mass-like lesions, and nonspecific white matter lesions (2-4). Because the angiotropic growth of this neoplasm mainly affects the small vessels, the magnetic resonance angiography (MRA) findings are usually normal (5). We herein report the case of an IVL patient who presented with diffuse cerebral vasoconstriction on MRA and a high serum myeloperoxidase-specific antineutrophil cytoplasmic antibody (MPO-ANCA) level.

\section{Case Report}

An 87-year-old woman who had developed fever and edema of the lower legs, which had persisted for three months, was admitted to our hospital. Other than being medicated for hypertension and hyperlipidemia for seven years, she had previously been well. Before this episode, she had been able to walk over short distances using a Zimmerframe; however, she was bedridden at the time of admission. A physical examination revealed that her body temperature was $36.0^{\circ} \mathrm{C}$ and her blood pressure was $118 / 76 \mathrm{mmHg}$. She had bilateral pitting edema of the lower legs. There were no

\footnotetext{
${ }^{1}$ Department of Neurology, Asahi General Hospital, Japan, ${ }^{2}$ Department of Neurology, Graduate School of Medicine, Chiba University, Japan, ${ }^{3}$ Research Center for Allergy and Clinical Immunology, Asahi General Hospital, Japan, ${ }^{4}$ Department of Hematology, Asahi General Hospital, Japan and ${ }^{5}$ Department of Clinical Pathology, Asahi General Hospital, Japan

Received for publication July 25, 2016; Accepted for publication October 28, 2016

Correspondence to Dr. Atsuhiko Sugiyama, chinneosyo0624@yahoo.co.jp
} 

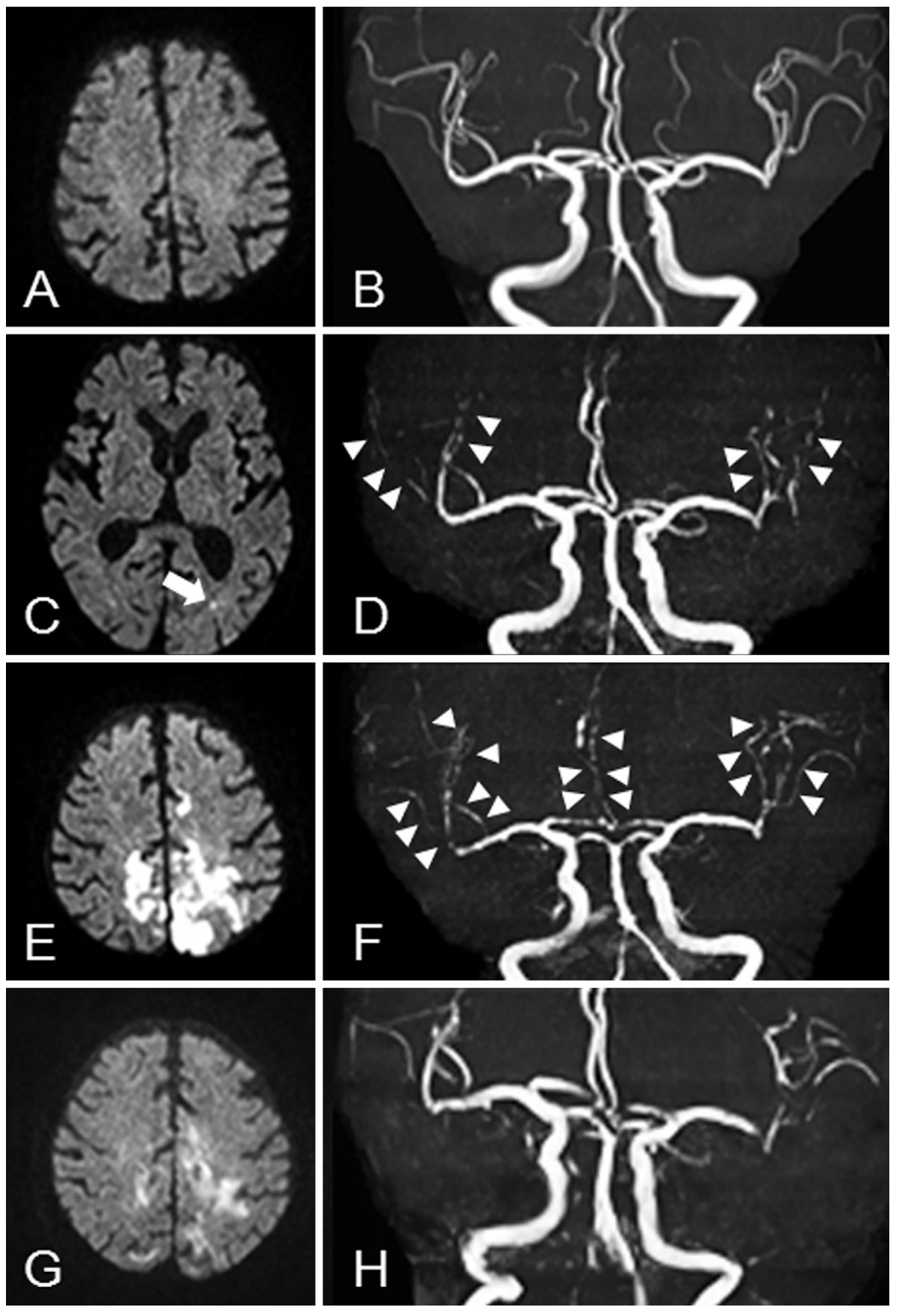

Figure 1. Brain magnetic resonance imaging (MRI) and magnetic resonance angiography (MRA). On admission, brain MRI (A) and MRA (B) were normal. On day 39, diffusion weighted imaging (DWI) showed a high intensity area in the left temporal lobe (white arrow) (C), and MRA revealed the bilateral diffuse narrowing of the M2 portion of the middle cerebral arteries (white arrowheads) (D). On day 49, DWI showed high-intensity areas in the left frontal lobe and bilateral parietal lobes (E), and MRA revealed the diffuse narrowing of the bilateral middle cerebral arteries, the anterior cerebral arteries, and the posterior cerebral arteries (white arrowheads) (F). After two cycle of R-CHOP therapy, the high intensity lesions on DWI had partially disappeared (G), and MRA showed the alleviation of the narrowing observed in the cerebral arteries $(\mathrm{H})$.

signs of superficial lymphadenopathy or cutaneous eruptions. A laboratory examination revealed the following findings: serum MPO-ANCA, $150 \mathrm{U} / \mathrm{mL}$ (reference range: $<3.50$ ); serum soluble IL-2 receptor, $1,570 \mathrm{U} / \mathrm{mL}$ (reference range: 145-519); serum CRP, $7.41 \mathrm{mg} / \mathrm{dL}$ (reference range: $<0.15$ ); and erythrocyte sedimentation rate, $113 \mathrm{~mm} / \mathrm{h}$ (refer- ence range: $<20$ ). A urinalysis revealed microscopic hematuria and proteinuria. Brain MRI and MRA revealed no abnormalities (Fig. 1A, B). A nerve conduction study revealed decreased action potentials in the bilateral tibial nerve and the right median nerve but failed to detect action potentials in the bilateral peroneal nerve, suggesting multiple 


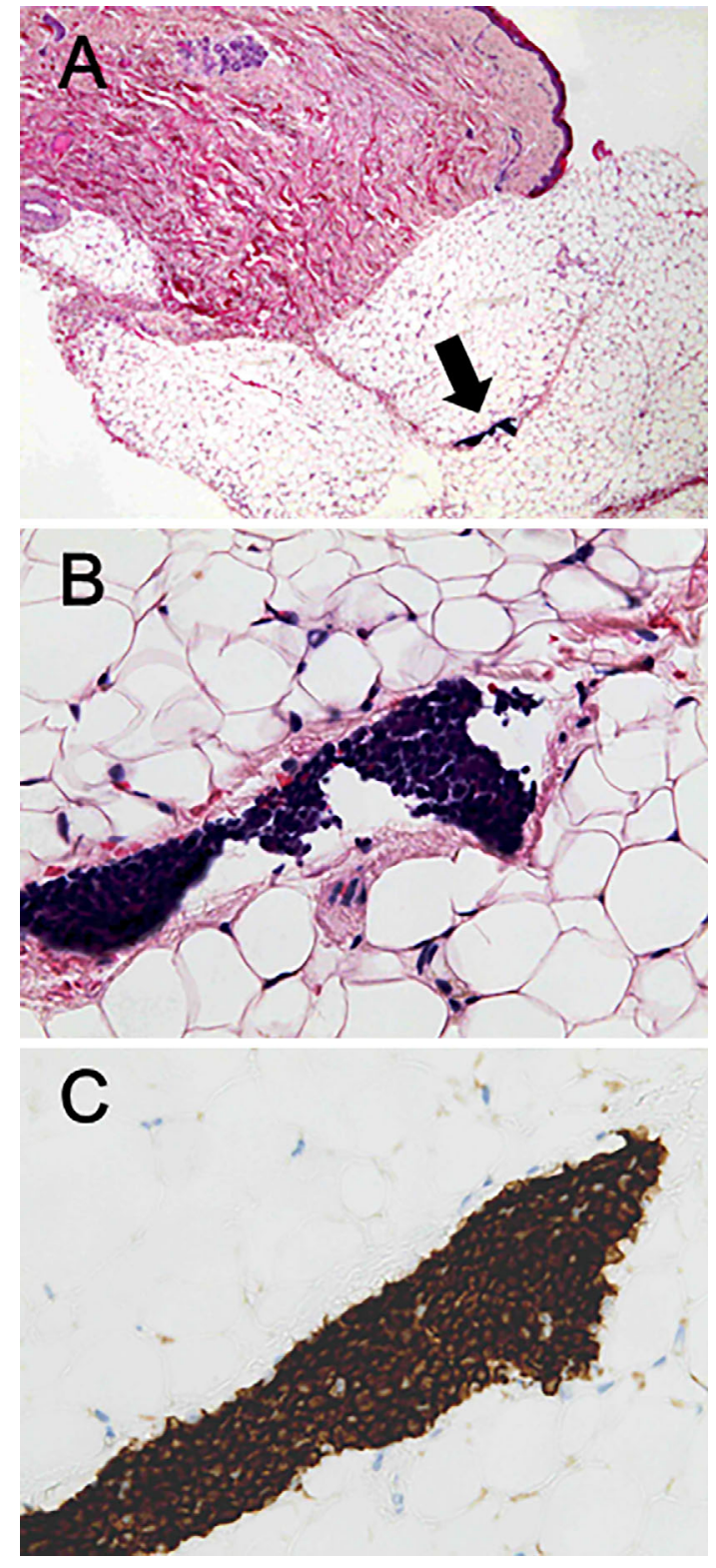

Figure 2. Skin biopsy. A histological specimen with Hematoxylin and Eosin staining, showing tumor cells in the lumen of the capillaries in subcutaneous fat (arrow) (A: low magnification, B: high magnification). The tumor cells in the capillaries were positively stained with a CD20 B-cell marker (C).

mononeuropathy. Microscopic polyangiitis (MPA) was suspected based on these findings, and treatment with prednisone $(45 \mathrm{mg} /$ day $)$ and rituximab $\left(375 \mathrm{mg} / \mathrm{m}^{2} /\right.$ week $)$ was initiated to prevent the further progression of MPA.

After the initiation of therapy, the inflammation subsided, and by day 32, the serum MPO-ANCA titer had decreased from 150 to $19.5 \mathrm{U} / \mathrm{mL}$. However, she developed transient hemiparesis of the right side on day 30. At that time, brain MRI revealed a small high-intensity lesion on diffusionweighted imaging (DWI) in the white matter of the left temporal lobe. MRA showed the bilateral narrowing of the distal middle cerebral arteries (MCA) (Fig. 1C, D). Thereafter, she gradually presented fluctuating disturbed consciousness. Her cerebrospinal fluid had a normal leukocyte count $(0 / \mathrm{mm}$ $\left.{ }^{3}\right)$ and an increased protein level $(62 \mathrm{mg} / \mathrm{dL})$. Brain MRI on day 49 described multiple hyperintense lesions on T2weighted imaging with diffusion restriction in the right cerebellum and both hemispheres (Fig. 1E). Focal hypertrophy and contrast enhancement in the pachymeninges of the right side convex was observed on T1-weighted images with contrast (not shown). MRA revealed the diffuse narrowing of the bilateral anterior cerebral arteries, the MCAs, and the posterior cerebral arteries (Fig. 1F). We performed random skin biopsies from the lower abdomen to investigate the cause of the infarct-like lesions and pachymeningeal involvement. The biopsy specimens showed intravascular large B cell lymphoma (Fig. 2).

Treatment with reduced-dose R-CHOP therapy (rituximab $375 \mathrm{mg} / \mathrm{m}^{2}$, doxorubicin $30 \mathrm{mg} /$ body, cyclophosphamide 500 $\mathrm{mg} /$ body, vincristine $0.8 \mathrm{mg} /$ body, and prednisolone $50 \mathrm{mg} /$ day) was immediately initiated. After two cycles of RCHOP brain MRI and MRA revealed the alleviation of vasoconstriction (Fig. 1G, H). After three cycles of RCHOP, she was transferred to another hospital.

\section{Discussion}

The MRA findings observed in our case were reversible, and were too diffuse and symmetric to have been caused by the direct invasion of lymphoma cells. We assume that the MRA findings in our case represented vasoconstriction, which occurred in the course of IVL. To the best of our knowledge, no cases of IVL with diffuse cerebral vasoconstriction have been reported; however, a case of IVL with diffuse pulmonary vasoconstriction has been described (6). This previous report assumed that the diffuse pulmonary vasoconstriction was caused by vasoactive mediators that were mainly released by activated platelets. Unfortunately, the previous report provided no clear evidence of the elevation of vasoactive mediators, and other mechanisms, including the direct interaction of lymphoma cells with endothelial cells might have caused the vasoconstriction. In IVL patients, endothelial injury might be caused by tumor-derived factors or by direct the interaction of lymphoma cells with endothelial cells. This endothelial damage leads to platelet activation and the formation of microthrombi (7), which can cause vasoconstriction. Drug-induced vasoconstriction is another possible cause of changes to the cerebral arteries. However, we considered this to be unlikely. The narrowing of the cerebral arteries was presumed to have started between the day of admission and day 30, and an improvement in the narrowing of the cerebral arteries was observed despite all of the drugs that were initiated during this period being continued.

The inflammatory reaction, the high serum MPO-ANCA titers, and multiple mononeuropathy at the initial evaluation of this case misled us into diagnosing the patient with MPA. Previous reports have indicated that several drugs can induce ANCA-associated vasculitis (8); however, these drugs were not prescribed to our case on admission. Cases of "false 
positive ANCA" have been reported in patients with several diseases such as HIV, tuberculosis, atrial myxomas/clot, and non-Hodgkin's lymphoma (9). In addition to the present case, there is only one other reported case of IVL in a patient with a high serum MPO-ANCA level (10). Under these conditions, vascular damage, due to immune-mediated mechanisms or to secondary to embolic damage appeared to predispose the patient to the development of ANCA antibodies (9).

In conclusion, diffuse cerebral vasoconstriction and a high serum MPO-ANCA level were observed in a patient with IVL. Endothelial damage caused by immune-mediated mechanisms, tumor-derived factors, or the direct interaction of lymphoma cells with endothelial cells may predispose a patient to both cerebral vasoconstriction and the development of ANCA antibodies.

The authors state that they have no Conflict of Interest (COI).

\section{References}

1. Fredericks RK, Walker FO, Elster A, Challa V. Angiotropic intravascular large-cell lymphoma (malignant angioendotheliomatosis): report of a case and review of the literature. Surg Neurol 35: 218-223, 1991.

2. Williams RL, Meltzer CC, Smirniotopoulos JG, Fukui MB, Inman M. Cerebral MR imaging inintravascular lymphomatosis. Am J Neuroradiol 19: 427-431, 1998.
3. Martin-Duverneuil N, Mokhtari K, Behin A, Lafitte F, Hoang-Xuan K, Chiras J. Intravascular malignant lymphomatosis. Neuroradiology 44: 749-754, 2002.

4. Yamamoto A, Kikuchi Y, Homma K, O'uchi T, Furui S. Characteristics of intravascular large B-cell lymphoma on cerebral MR imaging. Am J Neuroradiol 33: 292-296, 2012.

5. Jitpratoom P, Yuckpan P, Sitthinamsuwan $P$, Chotinaiwattarakul W, Chinthammitr Y. Progressive multifocal cerebral infarction from intravascular large B cell lymphoma presenting in a man: a case report. J Med Case Rep 5: 24, 2011.

6. Sekine A, Hagiwara E, Okudera K, Baba T, Ogura T. A case of slowly progressive intravascular lymphoma with respiratory failure caused by diffuse pulmonary vasoconstriction. Nihon Kokyuki Gakkai Zasshi 47: 924-929, 2009 (in Japanese).

7. Beristain X, Azzarelli B. The neurological masquerade of intravascular lymphomatosis. Arch Neurol 59: 439-443, 2002.

8. Gao Y, Zhao MH. Drug-induced antineutrophil cytoplasmic antibody-associated vasculitis. Nephrology (Carlton) 14: 33-41, 2009.

9. Davenport A. "False positive" perinuclear and cytoplasmic antineurtrophil cytoplasmic antibody results leading to misdiagnosis of Wegener's granulomatosis and/or microscopic polyarteritis. Clin Nephrol 37: 124-130, 1992.

10. Muto G, Takahashi Y, Yamashita H, Mimori A. A patient with intravascular lymphoma presenting with cerebral infarction and a high serum MPO-ANCA level. Mod Rheumatol 21: 207-210, 2011.

The Internal Medicine is an Open Access article distributed under the Creative Commons Attribution-NonCommercial-NoDerivatives 4.0 International License. To view the details of this license, please visit (https://creativecommons.org/licenses/ by-nc-nd/4.0/).

(C) 2017 The Japanese Society of Internal Medicine http://www.naika.or.jp/imonline/index.html 